\title{
THE 3-RAINBOW INDEX OF A GRAPH
}

\author{
Lily Chen $^{1}$, Xueliang Li ${ }^{1,2}$ \\ KANG YANG ${ }^{1}$ AND YAN ZHAO ${ }^{1}$ \\ Center for Combinatorics and LPMC-TJKLC \\ Nankai University \\ Tianjin 300071, China \\ e-mail: lily60612@126.com \\ lxl@nankai.edu.cn \\ yangkang@mail.nankai.edu.cn \\ zhaoyan2010@mail.nankai.edu.cn
}

\begin{abstract}
Let $G$ be a nontrivial connected graph with an edge-coloring $c: E(G) \rightarrow$ $\{1,2, \ldots, q\}, q \in \mathbb{N}$, where adjacent edges may be colored the same. A tree $T$ in $G$ is a rainbow tree if no two edges of $T$ receive the same color. For a vertex subset $S \subseteq V(G)$, a tree that connects $S$ in $G$ is called an $S$-tree. The minimum number of colors that are needed in an edge-coloring of $G$ such that there is a rainbow $S$-tree for each $k$-subset $S$ of $V(G)$ is called the $k$-rainbow index of $G$, denoted by $r x_{k}(G)$. In this paper, we first determine the graphs of size $m$ whose 3-rainbow index equals $m, m-1, m-2$ or 2 . We also obtain the exact values of $r x_{3}(G)$ when $G$ is a regular multipartite complete graph or a wheel. Finally, we give a sharp upper bound for $r x_{3}(G)$ when $G$ is 2-connected and 2-edge connected. Graphs $G$ for which $r x_{3}(G)$ attains this upper bound are determined.
\end{abstract}

Keywords: rainbow tree, $S$-tree, $k$-rainbow index.

2010 Mathematics Subject Classification: 05C05, 05C15, 05 C75.

\section{REFERENCES}

[1] J.A. Bondy and U.S.R. Murty, Graph Theory (GTM 244, Springer, 2008).

[2] Y. Caro, A. Lev, Y. Roditty, Zs. Tuza and R. Yuster, On rainbow connection, Electron. J. Combin. 15(1) (2008) R57.

\footnotetext{
${ }^{1}$ Supported by NSFC No.11371205 and 11071130 .

${ }^{2}$ Corresponding author.
} 
[3] G. Chartrand, G. Johns, K. McKeon and P. Zhang, Rainbow connection in graphs, Math. Bohem. 133 (2008) 85-98.

[4] G. Chartrand, F. Okamoto and P. Zhang, Rainbow trees in graphs and generalized connectivity, Networks 55 (2010) 360-367. doi:10.1002/net.20339

[5] G. Chartrand, G. Johns, K. McKeon and P. Zhang, The rainbow connectivity of a graph, Networks 54(2) (2009) 75-81. doi:10.1002/net.20296

[6] X. Li and Y. Sun, Rainbow Connections of Graphs (Springer Briefs in Math., Springer, 2012).

[7] X. Li, Y. Shi and Y. Sun, Rainbow connections of graphs: A survey, Graphs Combin. 29 (2013) 1-38.

doi:10.1007/s00373-012-1243-2

Received 27 December 2013

Accepted 12 February 2014 